\title{
Laboratory Methods for Evaluating the Effect of Low Level Laser Therapy (L LLT) In Wound Healing
}

\author{
${ }^{1}$ HAWKINS, D. AND ${ }^{1}$ ABRAHAMSE, $\mathrm{H}$. \\ ${ }^{1}$ Faculty of Health, University of Johannesburg, P.O. Box 17011, Doornfontein, \\ Johannesburg, South Africa, 2028.
}

Received: December, 2004

Accepted: January, 2005

\begin{abstract}
The basic tenet of laser therapy is that laser radiation has a wavelength dependent capability to alter cellular behaviour in the absence of significant heating. Low intensity radiation can inhibit as well as stimulate cellular activity. Laser therapy typically involves the delivery of $1-4 \mathrm{~J} / \mathrm{cm}^{2}$ to treatments sites with lasers having output powers between $10 \mathrm{~mW}$ and $90 \mathrm{~mW}$. There are two major areas of laser therapy research: the laboratory and the clinic. The laboratory presents the least ambiguous results. Here, although unsupported results do appear, the vast majority of published work finds clear evidence that laser irradiation alters cellular processes in a nonthermal, wavelength-dependent manner. Low energy laser irradiation alters he cellular function by effecting protein synthesis, cell growth and differentiation, cell motility, membrane potential and binding affinities, neurotransmitter release, ATP synthesis and prostaglandin synthesis. Laboratory findings provide scientific rati onale of laser therapy and the effect of laser therapy on cellular processes. This review outlines some of the current methods employed in the laboratory to measure the effect of low level laser therapy (LLLT) on cellular and molecular processes in the cell. This review briefly explains the different structural, cellular and molecular parameters and highlights some of the basic principles and protocols including specialized equipment requirements.
\end{abstract}

Keywords:
low level laser therapy, wound healing, laboratory, cellular, molecular

\section{INTRODUCTION}

The effects that optical radiation may have on tissue can be separated into categories depending on the portion of the spectrum (wavelength) that is incident on the tissue and the intensity (power density or irradiance) of the radiation. Wavelength is expressed in nanometers and irradiance is power delivered divided by area exposed (Basford, 1995).

Lasers used in laser therapy have output powers between 10 and $90 \mathrm{~mW}$ with associated temperature elevations $\leq 0.5-0.75^{\circ} \mathrm{C}$. The temperature changes are hardly noticeable and are far too low and localized to produce significant physiological effects. Low level laser treatment parameters include wavelengths of $632.8 \mathrm{~nm}, 820 \mathrm{~nm}, 830 \mathrm{~nm}$ or $904 \mathrm{~nm}$ with treatments ranging between $1-4 \mathrm{~J} / \mathrm{cm}^{2}$ for up to 30 s on a daily or alternate day schedule. Laboratory research suggests that radiant exposures $\leq 0.01 \mathrm{~J} / \mathrm{cm}^{2}$ can alter cellular processes. The reports by Mester et al.,(1971) stated that $1-4 \mathrm{~J} / \mathrm{cm}^{2}$ of laser irradiation induced healing of chronic nonhealing soft tissue ulcers and formed the start of clinical laser therapy (Mester et al, 1971). Laboratory work has provided some evidence and support for the use of LLLT in wound healing. Many investigators, although not uncontested, found hat visible and $\mathrm{IR}$ radiation stimulates capillary growth and granulation tissue formation and alters cytokine production. Other studies have shown altered keratinocyte motility and fibroblast movement following irradiation. Additional studies have found that laser irradiation may enhance, inhibit or have no effect on the function of cells. Improvements, particularly in the initial phase of wound healing, have been reported following laser irradiation in many rabbit and rodent experiments. The application of such findings to humans is still unclear since similar experiments with pigs, which have skin more similar to that of 
humans, have shown no benefit (Mester et al, 1971).

One conclusion is that laboratory studies support the concept that laser irradiation can modify cellular processes in a wavelengthdependent nonthermal manner. Another is that intensities sufficient to produce these effects on cells can be delivered to superficial joints and tissues typically treated with laser therapy (Gitomer and Jones, 1990).

The mechanisms of positive influence of LLLT are not completely understood. There are various local and systemic medico-biological processes that take place under the influence of LLLT. The action of laser light of optimal parameters has normalizing influence bringing to the total rising of adaptive potential and to the acceleration of treatment. Initial phase of any biological and clinical effect of LLLT is the absorption of quantum energy by biotissues with induction of primary photophysical and photochemical processes. The process of absorption is influenced by the optical properties of the biological object and the parameters of laser irradiation (Gasparyan, 2000).

There exists a strong relationship, first, between light parameters and biostimulation effects on a cellular level, and second, between the moment of irradiation and limits of magnitude of biostimulation effects. Systemic studies with cells clearly indicate that such parameters of light as wavelength, fluence, and intensity play the most important roles in both stimulation and inhibition of cellular metabolism (Karu, 1991).

In healing tissues the following sequence of events must take place. There must be an injury in the first place and then a haematoma is formed. Platelets are the first cell components to invade the wound site and initiate the wound healing process by releasing growth factors. Several chemicals are released by platelets and monocytes to attract fibroblasts to the haematoma. Inflammation occurs through the action of neutrophils, macrophages, and lymphocytes mediated by growth factors and proteases. Proliferation takes place through the action of fibroblasts and once they arrive at the site of injury, they begin to proliferate and synthesize collagen. Proliferation of fibroblasts, epithelial and endothelial cells is largely dependent on growth factors and collagen deposition. Remodelling is facilitated by collagen cross linking and collagen degradation increasing scar strength as maturation of scar formation occurs (Dyson, 1991).

Wound healing involves the following phases:

- Haemostasis: platelets, endothelial cells, fibrin and fibronectin act through growth factors and cytokines.

- Inflammation: the point when blood clots form, bacteria are attacked and there is an orderly recruitment of key cells into the wound site.
- Proliferation: when cells necessary for wound closure multiply at the wound site to make new tissue and blood vessels.

- Remodelling: when the wound is healed and the initial scar tissue is restructured.

Any device that can accelerate any of these processes (transition from haematoma to fibroplasias, the development of new blood vessels, the production of collagen, or even the remodelling process) could accelerate the healing process of wounds (Dyson, 1991). Literature indicates that laser photobioactivation accelerates inflammation, modulates the level of prostaglandin, enhances the action of macrophages, promotes fibroblast proliferation, facilitates collagen synthesis, fosters immunity and even accelerates the healing process. However, the following research questions should be considered: (1) does photobioactivation modulate healing (2) if so, what really happens to the tissue and (3) what are the ultrastructural, cellular or molecular changes induced by photobioactivation in tissue or cells (Dyson, 1991).

Growth factors are classified as cytokines, which are proteins that act as intracellular signals to allow cells to communicate with one another. Growth factors are actually a subclass of cytokines (non-antibody proteins released from some cells and act as mediators) that specifically stimulate the migration and proliferation of cells and synthesis of new tissues. The mechanism of action of growth factors relies on the interaction of the growth factor with the cell membrane. Growth factors are either autocrine (acting on the cell that produced them), juxtocrine (acting on an adjacent cell), paracrine (acting on the local environment), or endocrine (acting on a distant cell). Growth factors are involved in all phases of wound healing (Table 1) and also have the ability to regulate many other functions within the cell, including protein synthesis. Growth factors are essential to wound healing (Ortho-McNell Pharmaceutical, 2002). Growth factors attract useful cells and proteins to the wound, including immune cells to fight infection and other cells to form connective tissue. They stimulate and increase production of connective tissue, create a new supply of blood vessels to nourish the site and promote remodelling (Ortho-McNell Pharmaceutical, 2002).

Platelet derived growth factor is one of the earliest of several growth factors identified in the wound healing process. The growth factor is a product of platelets and other cell types as well, including macrophages and endothelial cells. The function of PDGF is most evident at the proliferation stage in open wounds where granulation begins. Upon release at the wound site, it stimulates migration and proliferation of cells important to the wound healing process (Dyson, 1991). 
Table 1:

Specific mediators that activate the cell type associated with each phase of wound healing ${ }^{7}$.

\begin{tabular}{|l|l|l|l}
\hline \multicolumn{1}{c|}{ Hemostasis } & Inflammation & Proliferation & Remodelling \\
\hline Platelets & Neutrophils & Fibroblasts & Collagen crosslinking \\
Growth factors & Macrophages & Growth factors & Collagen degradation \\
Cytokines & Growth factors & Collagen deposition & Proteases \\
Endothelial cells & Proteases & Epithelial cells & $\uparrow$ Scar strength \\
Cytokines & & Growth factors & $\downarrow$ Vascualrity \\
Fibrin & & Endothelial cells & \\
Fibronectin & & Growth factors & \\
\hline
\end{tabular}

Regulation of wound healing is by growth factors and cytokines that affect cell migration, proliferation and protein production. Cells enter the wound site by migration, which involves recognition of cell surface integrins and adhesion ligands on matrix proteins.

LLLT, when used in an appropriate manner, can stimulate the healing of injured tissues such as those of the dermis. Investigations into the mechanisms involved have shown that many of the types of cells whose interaction results in dermal repair can be affected in a therapeutically advantageous manner by treatment with LLLT both in vitro and in vivo. Mast cells and macrophages can be stimulated to release growth factors and other substances, while the proliferation of fibroblasts, endothelial cells and keratinocytes maintained in adverse conditions can also be stimulated (Dyson, 1991). The development of granulation tissue is mainly controlled by growth factors released from macrophages. Light of a number of different wavelengths, specifically $660,820,870 \mathrm{~nm}$, at an energy density of $2.4 \mathrm{~J} / \mathrm{cm}^{2}$, has been shown to stimulate the ability of macrophages in vitro to release growth factors, which stimulate fibroblast proliferation; in contrast, light of $880 \mathrm{~nm}$ wavelength was inhibitory (Smith, 1991). At $660 \mathrm{~nm}$ the stimulatory effect of LLLT is dose dependent for exposure to energy densities of $2.4-7.2 \mathrm{~J} / \mathrm{cm}^{2}$, the upper end of the range being most effective and with $9.6 \mathrm{~J} / \mathrm{cm}^{2}$ proving to be less effective than $7.2 \mathrm{~J} / \mathrm{cm}^{2}$.

One of the problems with some of the earlier studies by Mester et al., (1971) and a number of other studies performed in the earlier seventies is that there appear to be technical discrepancies and a lack of evaluative research tools (Enwemeka, 1991). For example, the use of photographic measurements to evaluate wound healing may be questioned on the grounds that such measurements do not provide any information about the actual depth of the wound. Since a deep wound with a small area may indeed be less healed than a larger shallow wound, photographic measurement techniques can yield misleading information. Another example states that mankind has achieved the ability to measure time in such small units as the nanosecond, the science of counting the number of days it takes a wound to heal completely can be questioned, just as the use of visual observation to ascertain healing can be judiciously queried. These methodological problems form doubts in the minds of many laser enthusiasts and researchers. It is for this reason that laboratory evaluation of wound healing using biochemical markers has become so necessary (Enwemeka, 1991).

\section{Evaluation of structural and functional changes}

Chemotaxis, defined as directed cell motion toward an extracellular gradient, plays an important role during fertilization, inflammation, wound healing and haematopoesis. The number of viable cells that have migrated through a special "chemotaxis" chamber usually determines the amount of chemotaxis (Rigau et al., 1995).

Experimental studies on animals and in vitro conditions provide evidence that $633 \mathrm{~nm}$ laser light can enhance wound healing. Rigau et al., (1995) studied the effects of the $633 \mathrm{~nm}$ laser on the behaviour and morphology of a primary fibroblast culture after a central scratch of 0.4$1 \mathrm{~mm}$ and two irradiations were performed ${ }^{10}$. The effect of the wounding and laser irradiation was studied using colony formation (formation of colonies in the central zone of the wound or on the edge of the scratch), haptotaxis (change of orientation of the edge cells) and chemotaxischemokinesis (movement or migration of cells across the wound). The number and intensity of colony formation, the haptotaxis of the edge fibroblasts and the fibroblasts present in the centre of the scratch were rated and the irradiated and non-irradiated controls compared. The group concluded that structural changes like colony formation, haptotaxis and chemotaxischemokinesis appear sooner in LLLT irradiated cultures than in non-treated controls. They also confirmed that LLLT induced fibroblast biological effects $^{10}$.

Our laboratory findings using wounded human skin fibroblasts (WS1) supported the findings of Rigau et al., (1995) and the fibroblasts displayed evidence of chemotaxis, colony formation and haptotaxis (Figure 1) which appeared sooner in cultures that were irradiated and incubated overnight at $37^{\circ} \mathrm{C}$.

Veselska and Janisch (2000) studied the effect of long wavelength ultraviolet (UV) radiation on the survival of L929 mouse 
fibroblasts and on the integrity of the two main cytoskeletal structures (microtubules and microfilaments). All cells were irradiated with an equal dose of UVA at $356 \mathrm{~nm}$, subsequently reincubated and collected at regular intervals for further processing. Cytoskeleton morphology observations, mtotic index assessment and cell viability tests were performed by fluorescence microscopy. The proliferating activity of cells after irradiation was measured by spectrophotometry using the neutral red assay. After short reparation periods (30 minutes to 3 hours) there was an increase in the number of apoptotic cells with typical features (plasma membrane blebbing and DNA fragmentation). The surviving cells were characterized by other changes in morphology (shrinking of normal cells or formation of giant multinuclear cells).

Flow cytometry can be used to detect morphological changes of cells. During apoptosis cells shrink and undergo an increase in granularity. This can be detected in the flow cytometer by the change in the light scatter properties of the cells. Cells are analysed by forward scatter (size) and side scatter (granularity). Healthy cells appear as large particles with a few smaller cells (apoptotic bodies) to the left of the main population. When cells undergo apoptosis there is an accumulation of small apoptotic bodies.

\section{Evaluation of cell viability and cytotoxicity}

One of the most common methods for measuring cell number or proliferation is the assessment of $\mathrm{TdR}$ incorporation using ${ }^{3} \mathrm{H}$ or BrdU. Other, colorimetric assays can be used to assess cell number, for example, the MTT colorimetric assay has been demonstrated to be extremely useful. Kato et al., (1999) used the MTT colorimetric assay and the trypan blue exclusion assay to assess cell viability and growth.

Cell viability is defined experimentally by the integrity of the outer cell membrane. If the cell membrane is full of "holes", it allows substances to move across the cell membrane that would normally be excluded. Vital dyes such as trypan blue or propidium iodide (DNA dye) are usually excluded from viable cells, but they can enter through "holes" in damaged cell membranes and stain the cytoplasm and therefore can be used as an indicator of nonviable cells (Riss et al, 2002).

Trypan blue (Sigma, T8154) is a polar dye that cannot cross intact cell membranes but crosses the membranes of necrotic cells and apoptotic cells undergoing secondary necrosis. It is therefore only partially informative in differentiating the mechanism of cell death but it is a useful, rapid and simple screening assay for viable cells. The $\%$ viability is defined as the number of viable cells $\div$ total number of cells $X$ 100. Cells undergoing the early stages of apoptosis have intact cell membranes and thus exclude polar dyes (Sambrook et al, 1989). The samples can also be examined for the morphological changes (blebbing) associated with apoptosis. The \% apoptosis is defined as the number of viable "blebbed" cells $\div$ total number of viable cells X 100 (Sambrook et al, 1989).

The MTT assay is a colorimetric assay that measures the reduction of (3-(4,5dimethylthiazol-2-yl)-2,5-diphenyltetrazolium bromide) by mitochondrial succinate dehydrogenase (Mossman, 1983). Since reduction of MTT (Sigma M5655) can only occur in metabolically active cells the level of activity is a measure of the viability of the cells. $200 \mu \mathrm{l}$ of cell suspension is incubated with $20 \mu$ of MTT reagent $(5 \mathrm{mg} / \mathrm{ml})$ for 1 hour at $37^{\circ} \mathrm{C}$ where the MTT enters the cells and passes into the mitochondria where it is reduced to an insoluble, formazen product. The cells are centrifuged at 2200 rpm for 5 minutes. The supernatant is removed and the pellet washed in PBS and centrifuged. The supernatant is removed and $100 \mu \mathrm{l}$ of isopropanol is added and the centrifuge tubes are mixed well to solubilize the formazen product. The absorbance is read at $550 \mathrm{~nm}$ (Mossman, 1983).

Kato et al., (1999) established a new method for assessing cell viability and cell growth. In this method, a cell lysis agent is combined with a fluorescent probe for nucleic acid, which exclusively passes through the disrupted membranes of dead cells but not intact membranes of viable cells. The distinctive feature of the probe is that it possesses a large fluorescence enhancement (460-fold) on binding to nucleic acid despite a very low intrinsic fluorescence. In this fluorometric assay, based on cell lysis and staining, the fluorescence intensity is linearly related to total cell number. To assess non-viable cell number the dye solution $(10 \mu l)$ is added to each well, and the plates are agitated on a plate shaker for 30 seconds. The fluorescence intensity is measured using a multiwell-plate fluorometer (excitation wavelength $420 \mathrm{~nm}$, emission wavelength $460 \mathrm{~nm})$. The results are similar to those obtained by MTT colorimetric assay and trypan blue exclusion assays. The main advantage is the ability to measure simultaneously both cell viability and cell growth rapidly.

Neutrophils play a major role in host defence via the phagocytosis and destruction of pathogens during acute inflammation (Chen et al, 1994). The binding of opsonized bacteria or immune complexes to neutrophil immunoglobulin receptors can activate a number of processes such as phagocytosis, degranulation and activation of the NADPH oxidase. Changes of intracellular $\mathrm{Ca}^{2+}$ and $\mathrm{pH}$ indicate cell injury.

Laser irradiation is assumed to accelerate the formation of a trans-membrane 
electrochemical proton gradient in mitochondria. This causes more $\mathrm{Ca}^{2+}$ to be released from the mitochondria to the cytoplasm by an antiport process, using the proton-motive force (pmf). At low laser doses, the additional $\mathrm{Ca}^{2+}$ transported into the cytoplasm (among other factors controlled by the pmf) triggers mitosis and enhances cell proliferation (Chen et al, 1994). At higher laser doses too much $\mathrm{Ca}^{2+}$ is released causing hyperactivity of $\mathrm{Ca}^{2+}$-ATPase, which exhausts the ATP reserves of the cell. Without the ATP energy reserves the cell-pump activity slows down and the ionic concentrations in the cells and the surrounding medium tend to equilibrate. Thus, due to intracellular proteins (when not compensated by macromolecules in the surrounding medium), the intramolecular osmotic pressure becomes larger than that of the medium. Water influx then produces cavities extruding from the cell walls. At still higher laser doses more $\mathrm{Ca}^{2+}$ influx completely exhausts the cell energy and the intracellular osmotic pressure explodes the cell.

During wound healing, release of adenosine triphosphate (ATP) from platelets potentially exposes the epidermis to concentrations of ATP known to alter cellular functions mediated via changes in inositol triphosphate (IP3) and intracellular calcium levels $\left(\mathrm{Ca}^{2+}\right)$. Unger et al., (1998) used quantitative time-lapse interference reflection microscopy (IRM) together with indirect alpha-beta-3 integrin immunofluorescence to measure the effects of extracellular calcium treatment on fractional leading edge adhesion after wounding. Injury derived intracellular calcium signals may regulate wound closure by an adhesion mediated mechanism (Unger et al, 1998).

Watson et al., (1997) studied the stimulation of intracellular calcium $\left(\mathrm{Ca}^{2+}\right)$ levels in human neutrophils by assessing the intracellular free $\mathrm{Ca}^{2+}$. Changes in intracellular $\mathrm{Ca}^{2+}$ concentration were monitored with a fluorescent probe Fluo-3. Fluo-3-AM (Sigma) is a cell permeable fluorescent indicator of intracellular calcium $\left(\mathrm{Ca}^{2+}\right)$ levels. It crosses the cell membrane due to its AM ester structure. Esterases in the cell then hydrolyse the AM ester to yield Fluo-3. This compound is non-fluorescent until associated with $\mathrm{Ca}^{2+}$. The lower binding affinity of this compound allows measurement of higher peaks of $\mathrm{Ca}^{2+}$ and has been used to detect photochemically generated cytosolic calcium pulses. It is often used as a fluorescent indicator for cytosolic $\mathrm{Ca}^{2+}$ based on rhodam ine and fluorescein chromphores. A detailed procedure for the use in measuring cytosolic $\mathrm{Ca}^{2+}$ in platelets and neutrophils in the presence of plasma has been reported. Briefly, $2 \times 10^{7}$ cells $/ \mathrm{ml}$ are loaded by incubation at $37^{\circ} \mathrm{C}$ for 30 minutes with $2 \mu \mathrm{M}$ Fluo-3-AM. The cells are washed twice and resuspended to $2 \times 10^{6}$ cells $/ \mathrm{ml}$ in $\mathrm{Ca}^{2+}$ free medium $(145 \mathrm{mM} \mathrm{NaCl}$, $1 \mathrm{mM} \mathrm{Na}_{2} \mathrm{HPO}_{4} \cdot 2 \mathrm{H}_{2} \mathrm{O}, 0.5 \mathrm{mM} \mathrm{MgSO}{ }_{4} \cdot 7 \mathrm{H}_{2} \mathrm{O}, 5 \mathrm{mM}$ glucose, $20 \mathrm{mM}$ Hepes, $\mathrm{pH} 7.4$ ) to which $1 \mathrm{mM}$ $\mathrm{CaCl}_{2}$ is added as required. Fluorescence is measured at $505 \mathrm{~nm}$ excitation and $530 \mathrm{~nm}$ emission.

The luminescent cell viability assay is a homogenous method of determining the number of viable cells in culture based on the quantitation of the ATP present, which signals the presence of metabolically active cells (Riss et al, 2002). CellTiter Glo ${ }^{\mathrm{TM}}$ luminescent cell viability assay can be used to monitor cytotoxicity as well as cell proliferation by measuring the number of viable cells in culture. The assay uses a unique, stable form of luciferase to measure adenosine 5'-triphosphate (ATP) as an indicator of metabolically active or viable cells to evaluate cytotoxicity. The presence of ATP is a useful marker of "life". The luciferase reaction requires ATP; conditions ensure that the amount of light produced is proportional to the amount of ATP present, reflecting the number of viable cells. The luminescent signal produced is proportional to the number of viable cells present in culture.

Briefly, an equal volume of reconstituted CellTiter-Glo reagent is added to 50 $\mathrm{\mu l}$ of cell suspension. The contents are mixed on an orbital shaker for 2 minutes to induce cell lysis. The contents are incubated at room temperature for 10 minutes to stabilize the luminescent signal and the luminescence signal is recorded in reading light units (RLU). A standard ATP curve is generated using 10 -fold serial dilutions of $1 \mu \mathrm{M}$ ATP in culture medium $(100 \mu \mathrm{l}$ of $1 \mu \mathrm{M}$ ATP solution contains $10^{-10}$ moles ATP(Crounch, 1993).

Metabolically active cells can also be detected with mitochondrial specific dyes. Rhodamine 123 (Rh123) is a cell-permeable, cationic fluorochrome that is actively sequestered into mitochondria without having any cytotoxic effects (Darzynkiewicz, 1982) . Uptake is rapid but the dye leaks out again if the cells are washed. Rh123 can be combined with propidium iodide $(\mathrm{PI})$ staining to differentiate live (Rh123 positive) from dead (PI positive) cells. Damage to the mitochondria results in reduced uptake of Rh123 and thus serves as an assay for mitochondria transmembrane potential. Rh123 can also be used to detect the early stages of oxidative stress and apoptosis in cells, which are associated with increased Rh123 fluorescence.

Fluorescein diacetate (FDA) is an uncharged fluorescent dye that can cross cell membranes and therefore enter intact cells. Once inside the cell it is rapidly esterified by the abundant esterases present in all cells. The product of this hydrolysis is a fluorescein, which is highly charges and cannot escape from live cells but can escape from necrotic (dead) cells. FDA staining can be combined with $\mathrm{PI}$ staining to identify live from dead cells (Hamori, 1980). 


$$
\begin{aligned}
\text { Lactate }+\mathrm{NAD}^{+}+\mathrm{H} & \Rightarrow \text { LDH } \\
\mathrm{NADH}+\mathrm{PMS} & \Rightarrow \mathrm{NAD}+\mathrm{PMS}-\mathrm{H} \\
\mathrm{PMS}-\mathrm{H}+\mathrm{TNBT} & \Rightarrow \mathrm{PMS}+\mathrm{TNBT}-\text { fomazen }
\end{aligned}
$$

PMS $=$ phenazine methosulphate $;$ TNBT $=$ tetranitroblue tetrazolium

Cell damage is associated with the leakage of intracellular, cytoplasmic contents and lactate dehydrogenase (LDH) and can be used as a reported molecule for this event. Lactate dehydrogenase, released from cell into culture medium is measured using a modification of a commercial kit (Sigma Chemical Co. Ltd) adapted for use in a 96 well format. This method is based on a series of linked enzyme reactions, the final reaction being the reduction of a tetrazolium salt to a coloured, insoluble, formazen product (Uney et al, 1993). The CytoTox $96{ }^{\circledR}$ non-radioactive cytotoxicity assay (Promega) is a colorimetric assay used for estimating the number of nonviable cells. The assay measures the release of lactate dehydrogenase (LDH), which is a stable cytosolic enzyme that is released into the culture medium from cells with a damaged membrane or upon cell lysis (Hoffman, 2002). LDH is measured by a coupled enzymatic assay that results in the reduction of a tetrazolium salt (INT) into a red formazen product. $100 \mu$ l of culture medium is removed and mixed with an equal volume of reconstituted substrate mix.

The plate is covered with foil and incubated at room temperature for 30 minutes, protected from light. $100 \mu \mathrm{l}$ of stop solution is added and absorbance is read at $490 \mathrm{~nm}$. The absorbance at $492 \mathrm{~nm}$ is directly proportional to the number of lysed cells. Maximum LDH release with complete cell lysis is induced by incubating the plate at $-70^{\circ} \mathrm{C}$ for approximately 30 minutes followed by thawing at $37^{\circ} \mathrm{C}$ for 15 minutes (Moravec, 1994).

Cells undergoing necrosis lose the integrity of their plasma membranes allowing material to leak out but also allowing polar dyes to enter the cell. In contrast cells undergoing the early stages of apoptosis have intact cells membranes and thus exclude polar dyes. This can be used to differentiate live and dead cells. Dyes that can be used include trypan blue and DNA dyes such as propidium iodide ( $\mathrm{PI})$. PI fluoresces red when it binds to DNA or double stranded RNA. For these assays the cells are not fixed, as this would allow the dye to enter all cells. PI staining is often combined with other staining methods to discriminate necrotic (dead) cells from apoptotic cells (Horan and Kappler, 1977).

\section{Evaluation of cell proliferation}

The proliferating activity of cell populations after irradiation can be determined by the neutral red assay (NRU) based on the ability of living cells to take up the neutral red dye from the medium and retain it in their lysosomes. The cells growing in 96-well microtitre plates are assessed immediately after irradiation or after reincubation. Approximately $5 \times 10^{4}$ cells in $100 \mu \mathrm{l}$ complete Eagle's minimal essential medium (EMEM) are incubated with $10 \%$ neutral red $\left(33 \mu \mathrm{g} / \mathrm{ml}\right.$ Sigma N2889) for 1 hour at $37^{\circ} \mathrm{C}$ to permit the uptake of the dye into the lysosomes, fixed with $1 \%$ formaldehyde for 30 minutes and solubilized with $1 \%$ acetic acid in $50 \%$ ethanol for 30 minutes. The optical density is measured using a microplate reader at $550 \mathrm{~nm}$ (Abe et al, 2001).

Pinheiro et al., (2002) used spectroscopy at $540 \mathrm{~nm}$ to measure cell proliferation or cell density of both irradiated and control samples. The optical density of $4 \times 10^{4}$ cells in $100 \mu \mathrm{l}$ complete EMEM is measured at $540 \mathrm{~nm}$.

Cell number is usually evaluated during in vitro studies to estimate metabolic or pharmacological effects of specific compounds (Garcia-Gasca, 2000). However, estimation of in vitro cell density by direct counting is a laborious and time consuming task, whereas indirect methods for cell quantitation have serious disadvantages such as environmental costs or inaccuracies derived from non-specific interferences. A new staining method for in vitro cell density quantitation was developed, which employs carmine (Sigma, C1022), a natural dye widely used for chromosome staining in cytological staining. Normal or transformed murine fibroblasts, avian normal fibroblasts, human epithelial HeLe cells and insect cells, inoculated at a range of cell densities $\left(1 \times 10^{3}\right.$ to $5 \times 10^{5}$ ), were fixed with $4 \%$ formaldehyde/PBS (phosphate buffered saline, $\mathrm{pH} 7.5$ ) and stained with $0.4 \%$ alcoholic- $\mathrm{HCl}$ carmine $(4 \mathrm{~g}$ of carmine in $15 \mathrm{ml}$ of bidistilled water with $1 \mathrm{ml}$ concentrated $\mathrm{HCl}$, boiled for 10 minutes with constant stirring. After cooling $100 \mathrm{ml}$ of $85 \%$ ethanol was added) for at least 2 hours. The stain retained in cell monolayers was extracted with $0.01 \mathrm{M} \mathrm{NaOH}$ and spectrophotometrically measured at $531 \mathrm{~nm}$. The carmine staining methods represents an easy, reliable, and precise alternative for in vitro cell quantitation, avoiding interferences and over a wide range of cell types and cell densities (Garcia Gasca, 2000) .

During cell proliferation the DNA has to be replicated before the cell is divided into two daughter cells. This dose association between DNA synthesis and cell doubling makes the 
measurement of DNA synthesis very attractive for assessing cell proliferation. If labelled DNA precursors are added to the cell culture, cells that are about to divide incorporate the labeled nucleotide into their DNA (Abe et al, 2001). Traditionally, those assays involve the use of radiolabelled nucleosides, particularly tritiated thymidine $\left(\left[{ }^{3} \mathrm{H}\right]-\mathrm{TdR}\right)$. The amount of $\left[{ }^{3} \mathrm{H}\right]-\mathrm{TdR}$ incorporated into the cellular DNA is quantitated by liquid scintillation counting (LSC). Experiments have shown that the thymidine analogue 5-bromo-2'-deoxy uridine (BrdU) is incorporated into cellular DNA like thymidine. The incorporated BrdU can be detected by a quantitative cellular enzyme immunoassay using monoclonal antibodies directed against BrdU. The use of $\mathrm{BrdU}$ for proliferation assays circumvents the disadvantages associated with the radioactive compound $[\mathrm{H}]-\mathrm{TdR}$. Abe et al., (1998) used the BrdU incorporation assay to detect DNA synthesizing cells. Incorporation of BrdU into newly synthesized DNA permits indirect detection of rapidly proliferating cells with fluorescently labelled anti-BrdU antibodies or certain nucleic acid stains, thereby facilitating the identification of cells that have progressed through the S-phase of the cell cycle during the BrdU labelling period. Cells were treated with $10 \mu \mathrm{mol} / \mathrm{L}$ bromodeoxyuridine (Sigma B5002)) for 30 minutes at $37^{\circ} \mathrm{C}$. After being processed for ALP cytochemical staining, cells were fixed and permeabilized with buffered formalin acetone for 1 minute, treated with $2 \mathrm{~mol} / \mathrm{L} \mathrm{HCl}$ for 20 minutes to denature DNA, and rinsed with $0.1 \mathrm{~mol} / \mathrm{L}$ borate buffer, $\mathrm{pH} 8.5$ for 2 minutes. Immunostaining was performed with anti-BrdU monoclonal antibody. Cells were regarded as positive when the nuclei showed a granular staining pattern.

Tritiated thymidine incorporation indicates proliferation of epidermal and dermal cells during reepithelialization and maintained viability after wounding (Kuwahara et al, 2000). Tritiated thymidine incorporation can be used in conjunction with increasing doses of platelet derived growth factor (PDGF-BB) and fibroblasts transfected with PDGF-BB gene to demonstrate DNA synthesis in diabetic and non-diabetic fibroblast lattices. The lattices contraction increases sgnificantly with increasing doses of PDGF-BB and fibroblasts transfected with the PDGF-BB gene when compared with control .

During wound healing and inflammation, fibroblasts express elevated ALP, which can be investigated by studying the ALP-inductive response of normal human fibroblasts to ascorbic acid (AsA). AsA induces ALP activity and protein in cells in conventional monolayer culture. The extracellular matrix environment regulates the induction of ALP expression by AsA in fibroblasts. The increase expression of alkaline phosphatase activity has been reported to be one of the phenotypic changes of fibroblasts in wounds and inflammation. Abe et al., (1998) investigated ALP expression using ALP cytochemical staining, cell counting, bromodeoxyuridine (BrdU) incorporation and ALP activity.

For the cytochemical staining, cells were fixed with $1 \%$ paraformaldehyde and stained for ALP activity with 5-bromo-4-chloro-3-indolyl phosphate (BCIP) and nitro blue tetrazolium (NBT) for 30 minutes at $37^{\circ}$. For the control, cells were stained with BCIP-NBT mixture in which $1 \mathrm{mmol} / \mathrm{L}$ of levamisole (an inhibitor of ALP), was added. The percentage of ALP positive cells was determined by examining from $500-700$ cells/well in triplicate. Cells with a strong, ring-like stain were regarded as ALP positive (Abe et al., 1998).

For the cell counting, aliquots of cell suspensions were mixed with trypan blue, and the number of viable cells counted with a haemocytometer. The number of viable cells was also determined by the neutral red assay.

Alkaline phosphatase (ALP) is a membrane bound enzyme released in inflammation, remodelling and cell proliferation and has been used as a marker for wound healing. ALP activity can be measured with a colorimetric assay using $\rho$-nitrophenyl phosphate as a substrate. 100ul of the culture medium is removed from each plate after each irradiation and preincubated with $100 \mu \mathrm{l}$ of $0.5 \mathrm{M} \mathrm{N}$-methylD-glucamine buffer, $\mathrm{pH} 10.5,0.5 \mathrm{mM}$ magnesium acetate, $110 \mathrm{mM} \mathrm{NaCl}$, and $0.22 \%$ Triton $\mathrm{X} 100$ for 30 minutes at $37^{\circ} \mathrm{C}$. $20 \mathrm{mM} \rho$-nitrophenyl phosphate ( $\rho$-NPP; Sigma) is added and the reaction was incubated at $37^{\circ} \mathrm{C}$ for 30 minutes. The amount of $\rho$-nitrophenol liberated is measured at $405 \mathrm{~nm}$ and the ALP activity expressed as nmoles $\rho-\mathrm{NP} / \mathrm{min} / 100 \mu$.

Neovascularization is essential for providing sufficient oxygen and nutrients and disposing of waste products from regenerating tissues and it plays an important role in wound healing. Alpaslan et al., (1997) evaluated the use of extracellular alkaline phosphatase activity as a marker of wound healing. The group studied the histopathologic aspects of the healing of fullthickness wounds created on the dorsal skin of rats by means of alkaline phosphatase (ALP) enzyme histochemistry and routine microscopy. Histochemical staining for ALP activity was performed at $4^{\circ} \mathrm{C}$ for 12 hours by the AZO dye method using Naphtol As -MX phosphate and Fast Red Violet TR salt as the substrate and capture reagent, respectively. The study showed an exclusive localization of extracellular ALP activity in the regions of granulation tissue formation. This localization was time related, and decreased as healing progressed. Extracellular ALP activity can be used as a simple and reliable histochemical process marker of skin wound healing.

The transmission of genetic information from one cell generation to the next requires the 
accurate duplication of the DNA during the $S$ phase of the cell cycle. Initiation of DNA replication is a highly regulated process that requires the ordered assembly of many proteins at the origin of DNA replication to form a competent, pre-replicative chromosomal state, as well as cell cycle regulated protein kinase pathways. Several proteins required for initiation are suspected to be pre-replicative components. Most strongly implicated is the initiation protein CDC6. CDC6 is a downstream target of molecular signalling pathways that control the $\mathrm{G}_{1} / \mathrm{S}$ transition. CDC6 is only expressed in actively replicating cells and quiescent cells in $\mathrm{G}_{0}$ do not express the protein, making it an excellent marker for cell proliferation. CDC6 immunolocalization may be used as an index of cell proliferation in tissue sections. Monoclonal antibody reacting specifically with CDC6 (Sigma, C0224) is also a useful tool in the investigation of the molecular mechanisms that determine how DNA replication is initiated, how it is restricted to certain cell cycle phases and how replication occurs in cells.

\section{Evaluation of growth factor expression}

Under pathological conditions such as wound healing and inflammation, morphological and functional features of fibroblasts are modulated by various environmental factors including growth factors, cytokines and extracellular matrix components.

An increase in cytokine expression is characteristic of accelerated wound closure and wound healing (Yeliseenko, 1994). Low level laser irradiation influences the activity of the mononuclear phagocytes (macrophages) by influencing the production of interleukin-1. The increase of this index is dependent on the irradiation dose and in optimal condition is mounted to $100 \%$. Mononuclear phagocytes produce factors that stimulate angiogenesis and fibrillogenesis and formation of granulation tissue in the areas. Flow cytometry assays of the mediators (interleukin-1, prostaglandin, component of complement) are used to show evidence of the above mechanisms. Proinflammatory cytokine release into media is quantitated by an ELISA assay to investigate the production of interleukin (IL-1 and IL-6) and chemokine IL-8 associated with inflammatory responses (Shear et al, 1999; Kramer et al, 2002).

Interleukin-6 (IL-6) is a pleiotropic cytokine produced by many cell types that acts on a wide range of tissues and cells, exerting growth inducing, growth inhibitory and differentiation induction effects depending on the nature of the target cells. The main producer cells in vivo are stimulated monocytes, fibroblasts and endothelial cells. It has also been shown that after stimulation $\mathrm{T}$ and B-lymphocytes, mast cells, glia-cells and keratinocytes are able to secrete IL-6. The IL-6 ELISA assay is an enzyme linked immunosorbent assay for the quantitative in vitro determination of biologically active IL- 6 in serum, cerebrospinal fluid and cell culture supernatants in streptavidin-coated microplates. The assay is based on the quantitative sandwich enzymeimmuno-assay principle, using two monoclonal antibodies from mouse, directed against two different epitopes of IL-6. Both antibodies recognize epitopes, which are essential for receptor binding. During the first incubation step, the biotin-labeled antibody simultaneously binds IL -6 in samples and the peroxidase conjugated detection antibody forming a complex, which binds via the biotin labelled antibody to the streptavidin-coated surface of the microplate. Subsequent to the washing step, the peroxidase bound in the complex is developed by tetramethylbenzidine (TMB) as a substrate and determined photometrically. The developed colour is proportional to the concentration of IL- 6 .

Fibroblast growth factors are potent regulators of cell proliferation, differentiation and are important in normal development, tissue maintenance and wound repair. The indirect ELISA has been used to evaluate the expression of basic fibroblast growth factor (bFGF) using a monoclonal anti-fibroblast growth factor primary antibody (Sigma-Aldrich F6162). Takamiya et al., (2003) studied the mRNA expression of basic fibroblast growth factor (bFGF) and found that time dependent expression of bFGF in the skin and cerebrum was considered to be useful for wound age determination. Lonergan et al., (2003) analysed the levels of bFGF in irradiated fibroblasts and concluded that cells from irradiated tissue undergo cellular changes and that fibroblasts exhibited stronger growth in modified Webber's medium (bFGF $50 \mathrm{ng} / \mathrm{ml}$ and insulin-like growth factor) than in Dulbecco's Modified Eagle Medium with 10\% fetal bovine serum (serum with undefined basal growth factors.

Briefly, an antigen solution containing approximately $1 \times 10^{5}$ cells in carbonatebicarbonate is incubated overnight at $4^{\circ} \mathrm{C}$ in a covered Nunc Maxisorp 96 -well microplate. The following morning the coating solution is removed and each well is washed three times with PBS-T (10mM phosphate buffer pH 7.4, $150 \mathrm{mM} \mathrm{NaCl}, 0.05 \%$ Tween 20 ). The primary antibody is diluted to a minimum titre of $5 \mu \mathrm{g} / \mathrm{ml}$ in PBS-T. 200 $\mu$ l of the diluted primary antibody is added to each well and the plate incubated at room temperature for 2 hours. Isotype matched, non-specific mouse immunoglobulin (Sigma, Mouse IgG Kappa) diluted in PBS-T can be used as a negative control. Each well is washed three times with PBS-T. The peroxidase-conjugated antibody is diluted in PBS-T and $200 \mu$ is added to each well and the plate incubated at room temperature for 2 hours. Each well is washed three times with PBS-T. 200 $\mu$ l of freshly prepared substrate for colour development is 
added to each well and the plate incubated for approximately 30 minutes. 5qul of stop solution $(3 \mathrm{M} \mathrm{HCl})$ is added to each well and the absorbance read at 492nm.

Vascular endothelial growth factor (VEGF) also known as vasculotropin, is an angiogenic growth factor which is heat and acid stable. VEGF is a dimeric, heparin-binding glycoprotein with a molecular weight of approximately $46 \mathrm{kDa}$. Briefly, in this bioassay 10ng/ml VEGF (Sigma, $\mathrm{V} 1253$ ) is incubated with various dilutions of the antibody for 1 hour at $22^{\circ} \mathrm{C}$ in a 96-well microtiter plate. After the preincubation the cells are added to the antigen-antibody mixture. The assay mixture is incubated at $37^{\circ}$ for 72 hours in a humidified $\mathrm{CO}_{2}$ incubator and pulsed for the final 24 hours with ${ }^{3} \mathrm{H}$-thymidine. Cells are harvested onto glass filters and the ${ }^{3} \mathrm{H}$-thymidine incorporation into DNA is measured. The antibody may also be used in immunoblotting and ELISA assay. In addition, the antibody does not cross react with other cytokines tested.

Several studies have shown that $\mathrm{He}-\mathrm{Ne}$ laser biostimulation, growth factors, and growth hormone can enhance cellular proliferation (AIWatban and Andres, 2000). VEGF is a potent mitogenic cytokine, which has been identified as the principal polypeptide growth factor influencing endothelial cell migration and proliferation. Progression of these two processes is an absolute prerequisite for initiating and maintaining the proliferative phase of wound healing. Systemic sepsis and wound contamination due to bacterial infection is associated with significant retardation of the proliferative phase of wound repair. Reverse transcriptase polymerase chain reaction and Northern blotting can analyze VEGF mRNA expression. Expression of vascular endothelial growth factor (VEGF) appears to be associated with the extent of neovascularization. VEGF receptor density on endothelial cells in the presence of lipopolysaccharide (LPS) and bacterial lipoprotein (BLP) can be assessed by flow cytometry to evaluate the repressive effects of LPS and BLP on the VEGF receptor in the relationship between bacterial sepsis, attenuated wound healing and VEGF receptor expression and proliferation (Power et al, 2001).

The effect of hepatocyte growth factor/scatter factor (HGF/SF) during wound healing in the skin can be investigated. Histological analysis of HGF/SF transgenic mouse excision wound sites reveal increased granulation tissue with marked vascularization (Toyoda et al, 2001). Norther $\mathrm{n}$ blot analysis demonstrates, relative to control, vascular endothelial growth factor (VEGF) expression in transgenic skin significantly higher at baseline and robustly up-regulated during wound healing. Elevated levels of VEGF protein are detected immunohistochemically, predominantly in endothelial cells and fibroblasts within the granulation tissue of HGF/SF transgenic skin. Serum levels of VEGF are also elevated in $\mathrm{HGF/SF}$ transgenic mice. Thus suggesting that $\mathrm{HGF} / \mathrm{SF}$ and the induction of VEGF has a significant effect on vascularization and granulation tissue formation during wound healing in vivo.

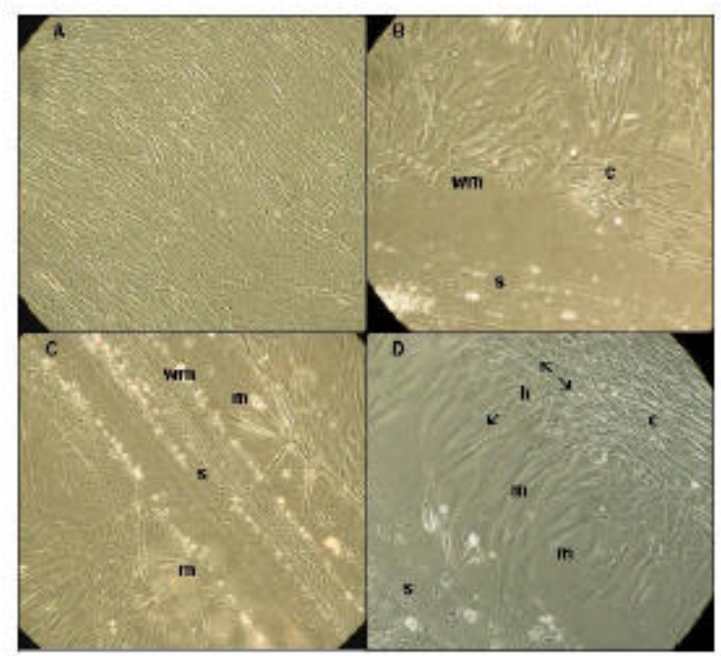

Plate 1

Normal human skin fibroblasts are normally long and slender and grow in monolayer sheets (A). For the simulated wounds (B, C, D) confluent monolayers were scratched with a sterile pipette (s). In the wounded fibroblast cultures the wound margin (wm) or edge could be clearly seen and cells appear in dense clusters (c) close to the edge (colony formation). Structural changes occur sooner in irradiated (C and $\mathbf{D}$ ) than in nonirradiated cultures $(\mathbf{B})$. The wound margin remains clearly defined in the non-irradiated control but irradiated cultures show evidence of cell migration across the wound edge. The irradiated cultures showed a greater rate of migration $(\mathrm{m})$ of the fibroblasts across the wound in an attempt to close the central scratch or wound. The occurrence of haptotaxis or change in the orientation of the edge cells (h) was more evident and occurred sooner in irradiated cultures than in non-irradiated controls. Irradiated cultures display more evidence of cell damage with detached and fragmented (lysed) cells 
mRNA of platelet derived growth factor (PDGF) receptor type alpha (PDGFR alpha) and type beta (PDGFR beta) are expressed in cultured corneal epinelial cells (CEP) during wound healing. The expression of mRNA and protein of both PDGF receptor subtypes in cultured CEPs and in CEPs ex vivo can be compared. Total RNA and protein are extracted from cultured CEPs and from CEPs ex vivo according to standard protocols. The reversetranscription polymerase chain reaction (RT-
PCR) is applied to detect specific expression of the mRNA of PDGFR alpha and PDGFRss. The expression of the corresponding proteins is detected using Western blotting (Denk and Knorr, 2002). mRNA and protein of both PDGF receptor subtypes can be detected in cultured CEPs but not in CEPs ex vivo. mRNA and protein of both PDGF receptor subtypes can be expressed in cultured human corneal epithelial cells, while corneal epithelial cells ex vivo do not express these receptors.

Table 2.

Summary of the tests used and the methods employed to measure the various effects of LLLT in vitro.

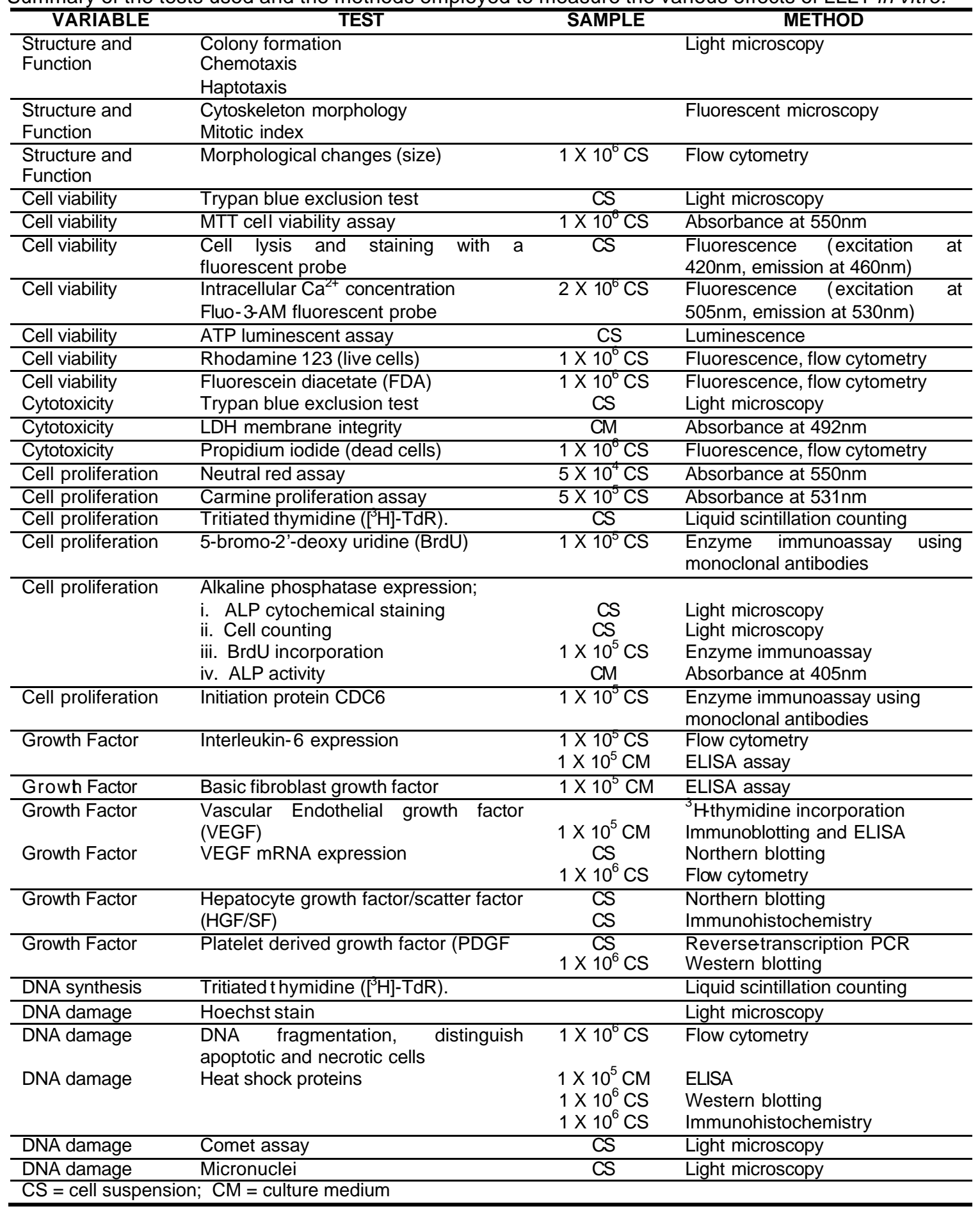


The differential up-regulation of PDGF receptors in activated cells during wound healing could be of pathophysiological importance (Denk and Knorr, 2002).

Variations in energy densities of LLLT produce differences in the proliferation of resting T-lymphocytes in vitro. The cells are pulsed with mitogen in addition to laser therapy to produce inhibitory effects regardless of the amplified of the energy density used. Isolated T-cells are prepared in suspension either with or without the addition of mitogen (Agaiby et al, 2000). Cell suspensions are irradiated with laser. After either 3 or 5 days incubation the lymphocyte supernatant is collected and added as conditioned media to cultured endothelial cells. The effects of the proliferation of these endothelial cells are assessed over a 72-hour period by using a methylene blue assay. Laser therapy stimulates lymphocytes to produce angiogenic factors that can modulate endothelial cell proliferation in vitro.

\section{Evaluation of DNA synthesis and DNA damage}

A research group from the Duquesne University in Pittsburgh studied the effect of UV light on baby hamster kidney fibroblasts. The cells were exposed to different energy levels of UV light with a frequency of $265 \mathrm{~nm}$ and $366 \mathrm{~nm}$ for different lengths of time. The cells were stained with trypan blue to observe cell death and Hoechst stain (only detects cell nuclei and DNA) to view nuclear breakdown. The results showed that the $366 \mathrm{~nm}$ wavelength caused more cell death than the $265 \mathrm{~nm}$ wavelength. The trypan blue showed a direct correlation between the percentage non-viable cells as the exposure time increased. Irradiated cells showed abnormalities of the cell nuclei, which included misshaped nuclei, nuclei that had exploded and DNA distributed throughout the cytoplasm. The group concluded that UV light could cause nuclei to become misshaped or lysed, which could lead to the distribution of DNA throughout the cytoplasm.

Flow cytometry can be used to detect DNA fragmentation using a DNA specific, fluorescent dye to measure the DNA content of individual cells ${ }^{43}$. Normal populations of growing cells have a single complement of DNA ( $G_{0}$ and $G_{1}$ phase), a double complement $\left(G_{2}\right.$ and $M$ phase cells) or an intermediate amount of DNA (S phase cells). This can be seen in the flow cytometer when the cells are labelled with a DNA specific dye such as propidium iodide ( $\mathrm{PI})$. Apoptotic cells contain fragmented DNA, which appears smaller than the $\mathrm{G}_{0}$ population. Cells are fixed to make them permeable to the PI dye and then analysed for red fluorescence in the flow cytometer. They are incubated with RNAase to remove RNA, which also binds PI (Nicoletti, 1991).
Heat shock proteins (Hsp) stabilize intracellular processes of cells under stress, are likely to be induced in the wound environment and may play a critical role in the overall process of wound repair (Souil et al, 2001). Skin biopsies are taken, the dermis and epidermis are separated and a protein lysate made. Western blotting and immunohistochemistry can be used to analyze the expression of Hsp 72, 47, and 32. In unwounded dermis, there was no constitutive expression of any of the heat shock proteins studied. In the epidermis, there is constitutive expression of Hsp 32 and 72, but not Hsp47. With wounding, all Hsps exhibit increased expression both in the dermis and epidermis. These patterns of protein expression are suggestive of the individual heat shock proteins' molecular function, such as Hsp 72's role as an indicator of cellular stress and injury, Hsp 47's role in collagen synthesis, and Hsp 32's role as an antioxidant.

Skin closure can be improved by photoirradiation of the wound margins. Expression of $\mathrm{Hsp} 70$ can be investigated by immunocytochemistry and compared with its expression after laser photoirradiation (Souil et al, 2001). Constitutive expression of Hsp70 is mainly confined to the upper epidermal layer. Laser irradiation increases epidermal expression of Hsp70 while inducing de novo synthesis of the protein in dermal structures, particularly around blood vessels, hair follicles and sebaceous glands. Laser-induced expression of Hsp70 might contribute to improved tissue regeneration and wound healing (Souil et al, 2001).

To examine the local heat shock response to wounding in diabetic mice tissues are analysed using immunohistochemical (IHC) and RT-PCR for inducible Hsp70. Hsp70 protein expression in the wound bed by IHC peaks at $24 \mathrm{~h}$ in the nondiabetic mice. Expression of Hsp70 is delayed in the diabetic mice, which correlates with the clinical delay in healing seen in this model $^{45}$. The protein is especially prominent in the epithelium and in inflammatory cells migrating into the granulation tissue matrix. RF PCR demonstrates upregulation of Hsp70 mRNA within $12 \mathrm{~h}$ after wounding, and decreasing thereafter in both the nondiabetic and the diabetic animals. Cutaneous wounding produces an Hsp response in inflammatory cells, and expression of inducible Hsp70 is delayed in diabetic mice. This delay may be related to the impaired inflammatory response of diabetics, and may contribute to impaired wound healing. The wound may be a continuing source of the heat shock response in inflammatory cells after injury (McMurtry et al, 1999).

Laser induced DNA damage can be studied using the Comet assay, a single cell technique used to detect DNA fragmentation in genomes ${ }^{46}$. Isolated cells are embedded in a $1 \%$ low melting temperature agarose and a drop placed on a 
microscope slide. Cells are lyzed in a non-ionic solution, which removes all of the proteins. The test can be made more specific and sensitive through the use of enzymes (Endo III and FPG). The lyzed cells are placed in an alkali solution where the damaged DNA relaxes and unwinds. The slides are placed in an electrophoretic tank for electrophoresis where the relaxed DNA is pulled out of the nucleoid towards the anode producing a comet like structure with the damaged DNA in the tail. The length and intensity of the tail corresponds to the amount of damage. The comets are neutralized and stained for scoring (Collins, 2000).

In testing for DNA damage, the micronuclei assay can be used as a biological assay for prescreening chromosome breakage. Micronuclei represent chromosomal fragments, which are lost from the nucleus during cell division but are retained in the cytoplasm. Previous results have shown that human skin fibroblasts exposed to laser irradiation show alterations in gene expression and micronuclei formation, an event that could eventually lead to phenotypic transformation in surviving cells (Samid et al., 1990). The micronuclei assay can be performed 72 hours after irradiation, the cells can be fixed with methanol/acetic acid and stained with propidium iodide following pretreatment with RNase. Micronuclei are scored by microscopic inspection of 500-800 cells in duplicate samples.

\section{CONCLUSION}

Depending on the extent of cellular death, wound healing occurs either by primary union (healing by first intention), or by secondary union (healing by second intention). The complex but systematic orchestrations of countless processes that govern wound healing have been extensively documented. Remarkable advances have been accomplished in our understanding and elucidation of the basic mechanism associated with wound healing. These include the contribution of mediators of acute inflammation and the role of growth factors in such processes as cell migration, cell proliferation and differentiation, angiogenesis, fibrosis and wound collagenization. Wound repair can be thought of as a culmination of the following three major overlapping events: inflammation, proliferation and remodelling. The sequencing and orchestration of the wound healing process is very closely regulated. A superb integration of dynamic reciprocity among cells, cytokines and matrix elements takes place (Aguirrer, 1997).

Basford (1995) concluded that laboratory studies support the concept that laser irradiation can modify cellular processes in a wavelength dependent, non-thermal manner and that intensities sufficient to produce these effects on cells can be delivered to the superficial joints and tissues typically treated with laser therapy ${ }^{1}$.
Laser therapy has proved to be effective and has the potential to move from the laboratory to the clinic. Whatever the clinical application of LLLT, whether it is tendonitis, arthritis, wound healing, pressure sores, dermatological conditions or chronic pain, the effects of the laser irradiation on the cells and tissue have to be established. The effects of LLLT include rapid cell growth, increased wound healing, increased metabolic activity, increased vascular activity and an anti-inflammatory action. These effe cts of LLLT have to be monitored in laboratory conditions and there are a number of established methods to evaluate the cellular and molecular effects of laser therapy on the cells. The parameters selected to evaluate the stimulatory or inhibitory effects of laser irradiation will depend on individual laboratory facilities (luminometer, flow cytometer and fluorometer) and the specific effect (structural, cellular or molecular) being evaluated (Table 2). Once structural, cellular and molecular effects of LLLT have been established and understood for a particular application (wound healing) the challenge will be to measure the same effects in an animal or clinical model using the same methods.

In literature most experiments use a single structural, cellular or molecular parameter to evaluate the effects of LLLT for a single application but these parameters can be used simultaneously to assess the general picture of the effect of LLLT on cells. The cell viability and cell proliferation studies can provide information about the metabolic activity, DNA synthesis and cellular damage while DNA damage and cytotoxicity (cell membrane integrity) studies can provide information about cellular and molecular damage to the cells. The growth factor studies can assess the intracellular signals that allow cells to communicate and can provide information during the different stages of wound healing namely haemostasis, inflammation and proliferation since they stimulate the migration and proliferation of cells and synthesis of new tissues. The combined use of all the biological parameters will be able to distinguish the different LLLT doses and whether the doses have a stimulatory or inhibitory effect on the cells. There are numerous methods available to the researcher however the final selection of the structural, cellular or molecular parameter depends on the specific effect that is being evaluated, the sample (tissue section or cell culture) and the laboratory facilities available. There are certain limitations (number of cells or suspension volume) to each method however some methods can be adapted to individual conditions allowing for a wide spectrum of tests on one sample.

In conclusion, laboratory methods can be used to assess the structural, cellular and molecular effects of LLLT on different cells resulting in reliable scientific results of the 
biological effects of LLLT so that LLLT may be used as an effective therapeutic modality for many medical applications.

\section{REFERENCES}

Abe T., Abe Y., Aida Y., Hara Y. and Maeda K. (2001) Extracellular matrix regulates induction of alkaline phosphatase expression by ascorbic acid in human fibroblasts. J Cell Physiol. 189 (2):144-151 Agaiby A.D., Ghali L.R., Wislon R. and Dyson M. (2000) Laser modulation of angiogenic factor production by T-lymphocytes. Lasers Surg Med. 26(4): 357-363

Aguirre, A. (1997) Extracellular alkaline phosphatase activity as a possible marker for wound healing: a preliminary report. J Oral Maxillofac Surg. 55: 62 63Al-Watban F.A. and Andres B.L. (2000) Effect of $\mathrm{He}-\mathrm{Ne}$ laser $(632.8 \mathrm{~nm})$ and Polygen on $\mathrm{CHO}$ cells. J. Clin. Laser Med. Surg. 18(3): 145-150

Aplaslan G., Nakajima T. and Takano Y. (1997) Extracellular alkaline phosphatase activity as a possible marker for wound healing: a preliminary report. Oral Mazillofac Surg. 55 (1): 56-62

Basford, J.R. (1995) Low Intensity Laser Therapy: Still not an established clinical tool. Lasers in Surgery and Medicine. 16: 331-342

Chen, T.S., Koutsilieri, E., Kruzik, P., and Rausch, W.D. (1994) Intracellular calcium and $\mathrm{pH}$ sensitive parameters of toxicity in neural cell culture. ALTEX.11 (4): $216-219$

Collins, A.R. (2000) Measurement of oxidative DNA damage using the Comet assay. In Measuring in vivo Oxidative Damage: A Practical Approach, ed. J. Lunec and H.R. Griffiths, pp. 83 - 94 John Wiley and Sons Ltd., England.

Crounch, S.P.M. (1993) The use of ATP bioluminescence as a measure of cell proliferation and cytotoxicity. Jour Immunol Meth. 160:81

Darzynkiewicz, Z. (1982) Detection of metabolically active cells with mitochondrial specific dyes. Cancer Research. 42: 799-806

Denk P.O and Knorr M. (2002) Differential regulation of expression of PDGF receptors on corneal epithelial cells. Ophthalmologe. 99(1): 15-19

Dyson, M. (1991) Cellular and Sub-cellular aspects of bw level laser therapy (LLLT). Progress in Laser Therapy: Selected papers from the October 1990 ILTA Congress. Published by Wiley and Sons, Inc. New York and Brisbane. 221,222

Enwemeka, C. (1991) Laser photostimulation in the United States - A tale of clinical tests, experimental trials, transient triumphs, and intermittent tribulations of a potential clinical armamenarium. Progress in Laser Therapy: Selected papers from the October 1990 ILTA Congress. Published by Wiley and Sons, Inc. New York and Brisbane. 103,104

Garcia-Gasca, T., Paz-Gonzalez, V., MoncadaAlvarez, M.C., Blanco-Labra, A., and Salazar-Olivo, L.A. Colorimetric quantitation of in vitro cell density using carmine, a chromosome specific stain. Toxicology in vitro. 16: 573-579

Gasparyan, L.V. (2000) Biochemical and Biophysical effects of Low Level Laser Irradiation. MAL Medical Acupuncture and Lasers Congress 28-30.9.2000, Helsinki, Finland.

Gitomer S.J. and Jones R.D. (1990) Laser produced plasmas in medicine. SPIE Vol. 1202, Laser-Tissue Interaction, ed. S.L.Jacques.
Hamori, E. (1980) Detection of live cells with fluorescein diacetate (FDA). Cytometry. 1: 132-135

Hoffman, R. (2002) In vitro toxicology and cellular fate determination using Promega's cell based assays. Promega Cell Notes. 4: 2-5

Horan, P.K., and Kappler, J.W. (1977) Dye exclusion assays (propidium iodide). J Immunol Methods. 18: 309-316

Karu, T. (1991) Low intensity laser light action upon fibroblasts and lymphocytes. Progress in Laser Therapy: Selected papers from the October 1990 ILTA Congress. Published by Wiley and Sons, Inc. New York and Brisbane. 175,

Kato, F., Tanaka, M., and Nakamura, K. (1999) Rapid fluorometric assay for cell viability and cell growth using nucleic acid staining and cell lysis agents. Toxicology. 13: 923-929

Kramer, G., Steiner, G., Handisurya, A., Stix, U., Haitel, A., Knerer, B., Gessl, A., Lee, L., and Marberger, M. (2002) Increased expression of lymphocyte derived cytokines in benign hyperplastic prostate tissue. Identification of the producing cell types, and effect of differentially expressed cytokines on stromal cell proliferation. The Prostate. 52: 43-58

Kuwahara H., Mitchell A.T., Macklim M.D., Zhao J., Listengarten D and Phillips L.G. (2000) Transfer of platelet derived growth factor-BB gene-by-gene gun increases concentration of collagen lattice by fibroblasts in diabetic and non-diabatic human skin. Scand J Plast Reconstr Surg Hand Surg. 34(4): 301307

Longergan, D.M., Mikulec, A.A., Hanasono, M.M., Kita, M., and Koch, R.J. (2003). Growth factor profile of irradiated human dermal fibroblasts using a serum free method. Plast Reconstr Surg. 111 (6): 1960-1968

McMurtryA.L., Cho K., Young L.J., Nelson C.F and Greenhalgh D.C. (1999) Expression of Hsp70 in healing wounds of diabetic and nondiabetic mice. $J$ Surg Res. 86(1) : 36-41

Mester, E., Spiry, T., Szende, B., Tota, J.G. (1971)

Effect of laser rays on wound healing. Am J Surg.122: 532

Moravec, R. (1994) Total cell quantitation using the CytoTox 96® non-radioactive cytotoxicity assay. Promega Notes. 45: 11-12

Mossman, T. (1983) The principle of the MTT assay. J. Immunol Methods. 65: 55-63

Nicoletti, I. (1991) Detection of DNA fragmentation by flow cytometry. J Immunlog Methods. 139: 271-279

Ortho-McNeil Pharmaceutical (2002), Regranex orbecaplermin gel product information

Pinheiro A.L.B., Nascimento S.C., Vieira A.L., Brugnera A., Zanin F.A., Rolim A.B. and Da Silva P.S. (2002) Effects of Low level laser therapy on malignant cells: In vitrostudy. J. Clin. Laser Med. and Surg. 20 (1): 23-26

Power, C, Wang, J.H., Sookhai, S., Street, J.T., and Redmond, H.P. (2001) Bacterial wall products induce downregulation of vascular endothelial growth factor receptors on endothelial calls via CD14 dependent mechanism: implications for surgical wound healing. J. Surg Res. 101 (2): 138-145

Rigau, J., Sun, C., Trelles, M.A., and Berns, M. (1995) Effects of the $633 \mathrm{~nm}$ laser on the behaviour and morphology of primary fibroblasts in culture. In proceedings, Effects of low power light on biological systems, Barcelona, Spain, eds T. Karu and A. Young, pp 38-42. Progress in Biomedical Optics, Barcelona, Spain

Riss, T., Moravec, R., Beck, M., Hannah, R., Wilson, K., and Swanson, R., (2002) CellTiter-Glo 
Luminescent Cell Viability Assay: Fast, Sensitive and Flexible. Promega Notes Magazine. 81: 2-5

Riss, T., Moravec, R., Beck, M., Hannah, R., Wilson, K., and Swanson, R., (2002) CellTiter-Glo Luminescent Cell Viability Assay: Fast, Sensitive and Flexible. Promega Notes Magazine. 81: 2-5

Rivera A.D.,, Walker C.N. Bleustein C., Choi B., Poppas D.P. and Felsen D. (2002) Enhanced adenoviral-vector mediated gene transfer using human albumin solder. Lasers Surg. Med. 30(4): 313319

Sambrook, J., Fritsch, E.F., and Maniatis, T. (1989) Molecular Cloning: A laboratory Manual, $2^{\text {nd }}$ Edition. Cold Spring Laboratory, Cold Spring Harbour, New York.

Shear, N.H., Landau, M., Malkiewicz, I., Katz, G.G. and Neuman, M.G. (1999) Ethanol-modulated cytokine production and expression in skin cells exposed to methotrexate. Skin Pharmacol Appl Skin Physiol. 12 (1-2): 64-78

Smith, K. (1991) Light and Life: The photobiological basis of the therapeutic use of radiation from lasers. Progress in Laser Therapy: Selected papers from the October 1990 ILTA Congress. Published by Wiley and Sons, Inc. New York and Brisbane. 14

Souil E., Capon A., Mordon S., Dinh Xuan AT., Polla B.S and Bachelet M. (2001) Treatment with $815 \mathrm{~nm}$ diode laser induces long lasting expression of $72 \mathrm{kDa}$ heat shock protein in normal rat skin. $\mathrm{Br} \mathrm{J}$ Dermatol. 144(2):260-266

Takamiya, M., Saigusa, K., Nakayashiki, N., and Aoki, Y. (2003) Studies on mRNA expression of basic fibroblast growth factor in wound healing for wound age determination. Int $J$ Legal Med. 117 (1):46-50

Toyoda M., Takayama H., Horiguchi N., Otsuka T., Fukusato T., Merlino G., Takagi H. and Mori M. (2001) Overexpression of hepatocyte growth factor/scatter factor promotes vascularization and granulation tissue formation in vivo. FEBS Lett. 509(1): 95-100

Uney, J.B., Anderton, B.H., and Thomas, S.M (1993) The principle of the Lactate dehydrogenase (LDH) assay. J Neurochemistry. 60: 659-665

Unger G.M., Bellrichard R.L., Trinh B.I. and Sammak P.G. (1998) Quantitative assessment of leading edge adhesion; reattachment kinetics modulated by injury derived intracellular calcium predict wound closure rates in endothelial monolayers. J Cell Physiol. 174(2): 217-231

Veselska, R. and Janisch, R. (2000) The effect of UV irradiation on changes in cytoskeleton and viability of mouse fibroblast L929 cell line. Scripta Medica (BRNO). 76: 393-408

Watson, F., Gasmi, L., and Edwards, S.W. (1997) Stimulation of intracellular calcium $\left(\mathrm{Ca}^{2+}\right)$ levels in human neutrophils by soluble immune complexes. Functional activation of FcgammaRlllb during priming. J Biol Chem. 272 (29): 17944 -17951

Yeliseenko, V.I. (1994) Low Level Lasers - their role in the mechanism of the stimulation of the reparative process. SPIE: Laser Tissue Interaction V. 2134A: 451-454

\section{Reprint requests}

Heidi Abrahamse (PhD), Senior Research Fellow, Witwatersrand. P.O. Box 17011, Doornfo ntein 2028 Gauteng, South Africa, Tel: +27 11 406-8145, Fax: +27 11 406-8202. e-mail: heidi@twr.ac.za 